\title{
Afetividade e Conflito Familiar e sua Relação com a Depressão em Crianças e Adolescentes
}

\author{
Affectivity and Family Conflict and their Relation with Depression \\ in Children and Adolescents
}

\author{
Maycoln L. M. Teodoro*, ${ }^{*}$, Bruna Moraes Cardoso ${ }^{b} \&$ Ana Carolina Huff Freitas ${ }^{b}$ \\ ${ }^{a}$ Universidade Federal de Minas Gerais \& ${ }^{b}$ Universidade do Vale do Rio dos Sinos
}

\begin{abstract}
Resumo
A presença de uma relação saudável da criança com seus pais é um fator importante na prevenção de psicopatologias como a depressão. O objetivo desta pesquisa foi investigar as propriedades psicométricas do Familiograma, associando os seus resultados de afetividade e conflito familiar com a intensidade da sintomatologia depressiva em crianças e adolescentes. Participaram do estudo 234 estudantes (133 do sexo feminino, 56,8\%) com idades entre oito a 14 anos (Média=11,19, DP=1,76). Os participantes responderam o Familiograma e o Inventário de Depressão Infantil. O Familiograma apresentou propriedades psicométricas satisfatórias. A depressão correlacionou-se negativamente com a afetividade $(r=-0,33)$ e positivamente com o conflito $(r=0,32)$. Os resultados apontam para a associação entre relações familiares pouco afetivas e conflituosas com a intensidade dos sintomas depressivos.

Palavras-chaves: Família; Relações familiares; Conflito familiar; Depressão.
\end{abstract}

\begin{abstract}
The presence of a healthy relation between a child and his/her parents is an important factor for the prevention of psychopathologies as depression. The objective of this research was to investigate the psychometrics properties of the Familiogram, and the association of the results of affectivity and family conflict with the intensity of depressive symptoms in children and adolescents. Two hundred thirty four students participated of the study (133 female, 56.8\%) and their ages ranged from 8 to 14 years old (Mean=11.19, $S D=1.76$ ). The participants answered the Familiogram and the Children Depression Inventory. The Familiogram presented satisfactory psychometrics properties. Depression correlated negatively with the affectivity ( $r=-0.33)$ and positively with the conflict $(r=0.32)$. Results point to the association of little affective and problematic family relations with the intensity of the depressive symptoms. Keywords: Family; Family relations; Family conflict; Depression.
\end{abstract}

A família inclui, em um sentido mais básico, parentes com ligações sanguíneas ou pessoas ligadas por casamento, união estável ou adoção, em que os membros cooperam economicamente, cuidam das crianças ou consideram sua identidade como sendo intimamente conectada (Bonomi, Boudreau, Fishman, Meenan, \& Revicki, 2005; Schneewind, 1999). Em um sentido mais amplo, este grupo incorpora a conexão com os indivíduos dentro de sua comunidade histórica, geográfica e sócio-cultural. Apesar de existir uma pluralidade na sua definição, é possível extrair do sistema familiar algumas características que o diferem de outros (veja maiores detalhes em

\footnotetext{
${ }^{*}$ Endereço para correspondência: Universidade Federal de Minas Gerais - FAFICH - Departamento de Psicologia, Av. Antonio Carlos, 6627, Pampulha, Belo Horizonte, MG, Brasil, CEP 31270-901.E-mail: mlmteodoro@hotmail.com Pesquisa realizada com apoio do Conselho Nacional de Desenvolvimento Científico e Tecnológico (Processo: 400337/2007-0).
}

Schneewind, 1999). O primeiro é a delimitação do espaço físico, no qual duas ou mais pessoas convivem no mesmo ambiente com regras explícitas e implícitas. Esta demarcação do espaço físico implica uma privacidade, na qual a troca comportamental é possível. Outra característica é a sua duração temporal, visto que o sentido de família está intimamente ligado a uma estabilidade ao longo do tempo. Finalmente, é necessária a presença de uma intimidade física e emocional no processo de relacionamento interpessoal.

O sistema familiar é reconhecido por vários teóricos como sendo de fundamental importância para a socialização primária e para a formação da identidade da criança. Como instituição, ela propicia à criança, e mais tarde ao adolescente, uma estrutura que garanta uma rede que promova apoio relacionado a problemas de diversos âmbitos, tais como sociais, escolares e financeiros (Pekrun, 2001; Rollett \& Werneck, 2002; Schneewind, 1999). Neste sentido, é importante a identificação dos aspectos 
do sistema familiar que estariam relacionados tanto ao bom funcionamento cognitivo e emocional de seus membros quanto a aspectos de psicopatologia.

A interação entre o relacionamento familiar e a intensidade de psicopatologia em seus membros vem sendo estudada por diversos pesquisadores. Fleitlich e Goodman (2001) e Hackett e Hackett (1999) encontraram que o estresse e doença mental nos pais estão positivamente associadas à presença de psicopatologias em crianças. $\mathrm{Da}$ mesma forma, a falta de disciplina, uso do castigo físico, desarmonia entre seus membros e, principalmente, a violência conjugal (Canino et al., 2004) são identificados como poderosos fatores de risco à saúde física e mental dos componentes do núcleo familiar (Goodman, Fleitlich-Bilyk, Patel, \& Goodman 2007; Hackett \& Hackett, 1999). Deste modo, alguns autores consideram famílias com relacionamentos adequados como sendo um fator de proteção para o aparecimento de futuras patologias infantis (Althoff, 2008; Feinberg, Button, Neiderhiser, Reis, \& Hetherington, 2007).

A presença de uma relação saudável da criança com seus pais é um dos fatores importantes na prevenção de psicopatologias, estando diretamente ligada à qualidade dos cuidados e das relações parentais (Dalton et al., 2006; Feinberg et al., 2007). Pedersen (1994), por exemplo, encontrou em uma amostra de crianças uma associação positiva entre a depressão e a percepção de baixo cuidado, principalmente pelo lado paterno. Resultados semelhantes foram descritos por Baptista e Oliveira (2004) em uma pesquisa desenvolvida no Brasil. Os autores encontraram que o suporte familiar inadequado, investigado na visão de adolescentes, estava negativamente associado com a depressão. Do mesmo modo, Galambos, Leadbeater, e Barker (2004) e Windle (1992) também encontraram que o fornecimento de suporte social pelos pais e pares aparenta ser um fator de proteção para a depressão.

Em um estudo longitudinal, Sheeber, Hops, Alpert, Davis, e Andrews (1997) investigaram as interações entre apoio, conflito familiar e sintomatologia depressiva na visão da mãe e do adolescente. Os resultados mostraram que ambientes familiares com mais conflito e menos suporte estavam associados a uma maior sintomatologia depressiva após um período de um ano. Por outro lado, a intensidade dos sintomas depressivos não atuava como preditor das relações familiares, sugerindo que a qualidade das interações familiares é relevante para a compreensão da evolução dos sintomas depressivos em adolescentes.

As interações familiares de baixa qualidade são tidas freqüentemente como um fator de risco para o aparecimento de sintomas depressivos. Existem, inclusive, evidências de que as relações familiares são preditores mais fidedignos do que as com pares na adolescência (McFarlane, Belíssimo, Norman, \& Lange, 1994). O estudo de preditores associados às relações sociais do indivíduo como fator de risco para a depressão é importante, na medida em que somente a contribuição genética da família não consegue explicar sozinha toda a variância desta psicopatologia (Sheeber, Hops, \& Davis, 2001).

Tendo em vista a importância da realização de estudos sobre o relacionamento familiar e suas associações com fatores de risco e proteção, torna-se essencial o desenvolvimento de instrumentos que avaliem estas relações e que possam ser utilizados dentro da realidade brasileira. Na literatura internacional, é corrente a publicação de artigos sobre o desenvolvimento e validação de instrumentos que avaliam as relações familiares, que são freqüentemente utilizados em pesquisas e/ou no cenário clínico (Bray, 1995). Por outro lado, no Brasil, existe ainda uma carência de instrumentos psicológicos nesta área.

Uma possibilidade de avaliação familiar é o Familiograma ([FG], Teodoro, 2005, 2006), que investiga dois conceitos teóricos. O primeiro, afetividade, foi definido como um conjunto de sentimentos positivos existentes entre as pessoas. $\mathrm{O}$ segundo, conflito familiar, foi caracterizado como uma gama de sentimentos que podem ser tanto uma fonte geradora de estresse como de agressividade dentro do sistema familiar. $\mathrm{O}$ instrumento consiste em uma lista de adjetivos que descrevem estes dois conceitos na díade familiar. Deste modo, o respondente informa para cada díade, a sua percepção sobre o nível de afetividade e conflito nas relações familiares.

Estudos com o Familiograma (Teodoro, 2005) investigaram a relação da coesão familiar com o construto de qualidade de vida em crianças brasileiras de nível socioeconômico médio. Os resultados mostraram que a coesão fraterna explicou cerca de $20 \%$ da variância da qualidade de vida destas crianças. Estes resultados confirmam pesquisas anteriores (Dundas, 1994; Phillips, West, Shen, \& Zheng, 1998) e apontam para a importância do sistema familiar na qualidade de vida de crianças. Reformulações na estrutura original do Familiograma foram apresentadas em Teodoro (2006). Neste estudo, foram investigadas algumas propriedades psicométricas da afetividade e do conflito em díades familiares na visão de universitários. Os resultados apontaram para uma estrutura bi-fatorial com consistência interna adequada.

Souza (2008) estudou a afetividade e conflito em famílias com pais alcoolistas na visão dos próprios pais, das esposas e dos filhos. Os resultados indicaram que as mulheres percebem a relação conjugal como sendo mais conflituosa do que as outras e uma afetividade maior com o filho em comparação com a díade paterna. Estes dados apontam para um distanciamento da relação afetiva entre o casal, sugerindo a presença de coalizões transgeracionais da mãe com o filho.

Tendo em vista o exposto, o presente estudo possui dois objetivos principais. O primeiro é explorar algumas propriedades psicométricas do Familiograma em crianças e adolescentes. $\mathrm{O}$ segundo é investigar, na visão dos filhos, a presença de correlações entre relações familiares (afetividade e conflito) e a intensidade dos sintomas da depressão. 


\section{Método}

\section{Amostra}

Participaram do estudo 234 estudantes de três escolas públicas da grande Porto Alegre selecionadas por conveniência. A amostra foi composta por 133 participantes do sexo feminino $(56,8 \%)$ e 101 do sexo masculino $(43,2 \%)$. A idade variou de oito a 14 anos (Média=11,19, $D P=1,76)$. Dentre as crianças e adolescentes participantes, 150 moravam com o pai e a mãe $(64,1 \%$ com configuração nuclear) e 84 moravam com apenas um ou nenhum dos pais $(35,9 \%$, configuração não-nuclear).

\section{Instrumentos}

Familiograma (Teodoro, 2006). O Familiograma avalia a percepção da afetividade e o conflito familiar nas díades familiares. Neste estudo foram avaliados, na visão das crianças e adolescentes, dois grupos de díades. No primeiro estão aquelas nas quais o participante interage: participante-mãe, participante-pai e participanteirmão. O segundo grupo expressa a percepção externa do participante com relação às díades pai-mãe, mãeirmão e pai-irmão. Além dos escores das díades pesquisadas, foi calculado um escore familiar para afetividade e outro para conflito que consiste no escore médio obtido para as díades estudadas.

Para preencher o instrumento, o participante precisava descrever para cada díade como era o relacionamento a partir de 22 adjetivos, em uma escala Likert de cinco pontos (variando de "de jeito nenhum" até "completamente"). Para o construto afetividade, foram usados os seguintes adjetivos: carinhoso, alegre, agradável, verdadeiro, afetivo, protetor, amoroso, acolhedor, harmonioso, atencioso, precioso. O conflito foi avaliado por meio das seguintes palavras: confuso, nervoso, estressante, baixo-astral, ruim, sufocante, tenso, frio, difícil, agressivo, chato. Os adjetivos foram selecionados a partir de uma lista de descritores de personalidade da língua portuguesa (Pinho, 2005) e dos resultados contidos em Teodoro (2006).

A pontuação do Familiograma varia de 11 a 55 para cada construto, sendo que quanto maior for o escore, maior será a percepção de afetividade e conflito. O Familiograma permite a classificação das famílias em quatro diferentes categorias de acordo com a intensidade da afetividade e do conflito familiar (vide Figura 1). As famílias pertencentes ao Tipo I são aquelas descritas como tendo alta afetividade e baixo conflito. Famílias do Tipo II possuem alta afetividade e alto conflito. Já as famílias classificadas como Tipo III possuem baixa afetividade e baixo conflito, enquanto as do Tipo IV possuem baixa afetividade e alto conflito.

Em um estudo psicométrico, Teodoro (2006) demonstrou a existência de uma estrutura bi-fatorial e Alfas de Cronbach variando de 0,87 até 0,97 para o Familiograma. No estudo atual, as análises de consistência interna foram de 0,89 (afetividade) e 0,87 (conflito) para a díade

\begin{tabular}{|c|c|}
\hline $\begin{array}{c}\text { Tipo I } \\
\text { Alta afetividade } \\
\text { Baixo conflito }\end{array}$ & $\begin{array}{c}\text { Tipo II } \\
\text { Alta afetividade } \\
\text { Alto conflito }\end{array}$ \\
\hline $\begin{array}{c}\text { Tipo III } \\
\text { Baixa afetividade } \\
\text { Baixo conflito }\end{array}$ & $\begin{array}{c}\text { Tipo IV } \\
\text { Baixa afetividade } \\
\text { Alto conflito }\end{array}$ \\
\hline
\end{tabular}

Figura 1. Classificação de tipos de família de acordo com os construtos afetividade e conflito do Familiograma

participante-mãe, 0,90 (afetividade) e 0,89 (conflito) para a díade participante-pai e 0,91 (afetividade) e 0,89 (conflito) para a díade participante-irmão. Para o segundo grupo de díades, os Alfas foram de 0,93 (afetividade) e 0,92 (conflito) para a díade mãe-pai, 0,89 (afetividade) e 0,90 (conflito) para a díade mãe-irmão e 0,90 (afetividade) e 0,89 (conflito) para a relação pai-irmão (vide Tabelas 1 e 2). Estes resultados indicam que o instrumento manteve a consistência interna na amostra originalmente estudada.

Inventário de Depressão Infantil ([CDI], Gouveia, Barbosa, Almeida, \& Gaião, 1995). O CDI investiga a intensidade dos sintomas de depressão em crianças e adolescentes. O instrumento é composto por 27 itens com três opções de resposta. Os participantes precisam escolher a alternativa que melhor descreva o seu estado emocional na atualidade. O CDI foi adaptado para uso no Brasil em um estudo realizado com 305 estudantes de João Pessoa (Gouveia et al., 1995). A versão adaptada apresentou consistência interna de 0,81 , semelhante ao encontrado por Wathier, Dell'Aglio, e Bandeira (2008) em uma amostra de 951 crianças e adolescentes em Porto Alegre $(A l f a=0,85)$. No presente estudo foi encontrado um Alfa de Cronbach de 0,80 para a escala.

\section{Procedimentos Éticos e de Pesquisa}

Este estudo faz parte de um projeto maior de avaliação familiar desenvolvido pelos autores. A pesquisa foi aprovada pelo Comitê de Ética da Universidade do Vale do Rio dos Sinos (Processo 06/032) e seguiu todas as recomendações éticas necessárias.

Os contatos com os participantes foram feitos por meio da diretoria da escola. Os estudantes receberam uma explicação sobre os objetivos da investigação e, aqueles que se interessaram, levaram o Termo de Consentimento Livre e Esclarecido para ser assinado pelos pais ou responsáveis. A avaliação foi feita coletivamente para alunos com mais de 10 anos, e individualmente para os menores. O tempo médio da aplicação foi de aproximadamente 20 minutos.

\section{Análise dos Dados}

As estatísticas foram realizadas em três etapas. A primeira consistiu de algumas análises psicométricas do Familiograma por meio de análises fatoriais explorató- 
rias e confirmatórias e de consistência interna. Para a análise exploratória foi utilizado como critério de seleção do número de fatores o gráfico de sedimentação. A análise foi feita com o método dos eixos principais (Principal Axis Factoring) e rotação Oblimin. Foi realizada uma análise fatorial para cada díade pesquisada (participante-mãe, participante-pai, participante-irmão, mãe-pai, mãe-irmão e pai-irmão). Calcularam-se também os índices de consistência interna por meio do Alfa de Cronbach. A análise confirmatória foi realizada com programa Lisrel 8.8, com o método de estimação Maximum Likelihood. Foram selecionados quatro indicadores de adequação do modelo para analisar os resultados da análise (maiores detalhes em Byrne, 1989). O primeiro é a razão $X^{2} / g l$, para o qual valores inferiores a 5 podem ser interpretados como um indicativo de que o modelo teórico se ajusta aos dados. O segundo é o Goodness-of-Fit Index (GFI), que deve ter escores iguais ou superiores a 0,90. Estes valores devem ser também considerados para o terceiro índice analisado, o Adjusted Goodness-of-Fit Index (AGFI). O último escore é o Root Mean Square Error Approximation (RMSEA), que deve ter valores até 0,10 para a aceitação do modelo. RMSEA inferiores a 0,05 indicam ótimos ajustes. Estes índices avaliam tanto a adequação do modelo teórico aos dados, quanto a proporção de variância-covariância explicada.
A segunda etapa da investigação dos dados foi composta de análises comparativas dos construtos afetividade e conflito. Diferenças e semelhanças da afetividade e conflito com as variáveis demográficas (sexo, idade e estrutura de família) foram realizadas por meio de teste $t$ e Correlação de Pearson. A comparação da intensidade dos construtos entre as díades foi feita por meio de Análise de Variância para Medidas Repetidas. Finalmente, a terceira etapa consistiu em comparações entre os escores de afetividade e conflito familiar e depressão, por meio de Correlação de Pearson e Análise de Variância OneWay com análises post hoc de Bonferroni. O nível de significância utilizado para as análises foi de $p<0,01$.

\section{Resultados}

\section{Propriedades Psicométricas do Familiograma}

Análises fatoriais exploratórias com o método dos eixos principais e rotação Oblimin foram realizadas para cada díade pesquisada. A apresentação dos resultados foi agrupada de acordo com o fato das crianças e adolescentes participarem da díade (participante-mãe, participante-pai e participante-irmão) ou serem observadores da relação (mãe-pai, mãe-irmão e pai-irmão). Os resultados indicaram a existência de uma estrutura bifatorial, semelhante para todas as díades estudadas (vide

Tabela 1

Análises Fatoriais do Familiograma com as Respectivas Cargas Fatoriais, Alfas de Cronbach e Variância Explicada para as díades Participante-Mãe (n=217), Participante-Pai $(n=200)$, Participante-Irmão $(n=183)$

\begin{tabular}{|c|c|c|c|c|c|c|}
\hline \multirow{3}{*}{ Itens } & \multicolumn{5}{|c|}{ Cargas Fatoriais } & \\
\hline & \multicolumn{2}{|c|}{ Participante-Mãe } & \multicolumn{2}{|c|}{ Participante-Pai } & \multicolumn{2}{|c|}{ Participante-Irmão } \\
\hline & Afetiv. & Conflito & Afetiv. & Conflito & Afetiv. & Conflito \\
\hline acolhedor & 0,64 & & 0,61 & & 0,69 & \\
\hline afetivo & 0,61 & & 0,62 & & 0,64 & \\
\hline agradável & 0,60 & & 0,72 & & 0,66 & \\
\hline alegre & 0,63 & & 0,67 & & 0,71 & \\
\hline amoroso & 0,79 & & 0,61 & & 0,75 & \\
\hline atencioso & 0,68 & & 0,70 & & 0,74 & \\
\hline carinhoso & 0,59 & & 0,62 & & 0,70 & \\
\hline harmonioso & 0,65 & & 0,70 & & 0,68 & \\
\hline precioso & 0,66 & & 0,74 & & 0,69 & \\
\hline protetor & 0,66 & & 0,69 & & 0,82 & \\
\hline verdadeiro & 0,62 & & 0,71 & & 0,67 & \\
\hline Agressivo & & 0,62 & & 0,65 & & 0,66 \\
\hline baixo-astral & & 0,47 & & 0,69 & & 0,55 \\
\hline chato & & 0,66 & & 0,58 & & 0,71 \\
\hline confuso & & 0,41 & & 0,67 & & 0,54 \\
\hline difícil & & 0,68 & & 0,77 & & 0,74 \\
\hline estressante & & 0,71 & & 0,69 & & 0,58 \\
\hline frio & & 0,68 & & 0,54 & & 0,76 \\
\hline nervoso & & 0,56 & & 0,69 & & 0,62 \\
\hline ruim & & 0,60 & & 0,65 & & 0,63 \\
\hline sufocante & & 0,55 & & 0,61 & & 0,65 \\
\hline tenso & & 0,66 & & 0,66 & & 0,68 \\
\hline Alfa de Cronbach & 0,89 & 0,87 & 0,90 & 0,89 & 0,91 & 0,89 \\
\hline Variância Explicada & \multicolumn{2}{|c|}{$42,00 \%$} & \multicolumn{2}{|c|}{$46,67 \%$} & \multicolumn{2}{|c|}{$48,55 \%$} \\
\hline
\end{tabular}


Tabelas $1 \mathrm{e}$ 2). Em todas as análises foram encontrados os fatores afetividade e conflito. $\mathrm{O}$ construto afetividade é composto pelo somatório dos escores dos adjetivos acolhedor, afetivo, agradável, alegre, amoroso, atencioso, carinhoso, harmonioso, precioso, protetor e verdadeiro. O fator conflito é a soma dos escores atribuídos para agressivo, baixo-astral, chato, confuso, difícil, estressante, frio, nervoso, ruim, sufocante e tenso.

Na Tabela 1 estão descritos os resultados das análises fatoriais das díades das quais os participantes fazem parte. Os resultados para os dois fatores explicaram de $42 \%$ a $48,55 \%$ da variância para a estrutura bi-fatorial. As análises do teste de esfericidade de Bartlett foram significativas para as díades participante-mãe $\left(X^{2}=1886,86\right.$; $g l=231)$, participante-pai $\left(X^{2}=2101,49 ; g l=231\right)$ e para a díade participante-irmão $\left(X^{2}=2130,16 ; g l=231\right)$. Com relação aos índices de Kaiser-Meyer-Olkin (KMO) de adequação da amostra, foram encontrados escores satisfatórios para as díades participante-mãe e participantepai $(\mathrm{KMO}=0,90)$ e para a díade participante-irmão $(\mathrm{KMO}=0,91)$. Análises de consistência interna, realizadas por meio do Alfa de Cronbach, apresentaram escores variando de 0,87 a 0,93 , sugerindo a presença de índices de fidedignidade adequados para os construtos.

As análises com as díades das quais os participantes não fazem parte diretamente, mas atuam como observadores, são apresentadas na Tabela 2. As análises de esfericidade de Bartlett foram significativas para as díades mãe-pai $\left(X^{2}=2856,27 ; g l=231\right)$, mãe-irmão $\left(X^{2}=1687,62\right.$; $g l=231)$ e pai-irmão $\left(X^{2}=1561,60 ; g l=231\right)$. O resultado dos escores de Kaiser-Meyer-Olkin (KMO) de adequação da amostra apresentou escores satisfatórios para as díades mãe-pai $(\mathrm{KMO}=0,93)$, mãe-irmão $(\mathrm{KMO}=$ $0,86)$ e pai-irmão $(\mathrm{KMO}=0,86)$.

Tabela 2

Análises Fatoriais do Familiograma com as Respectivas Cargas Fatoriais, Alfas de Cronbach e Variância Explicada para as díades Mãe-Pai (n=192), Mãe-Irmão (n=152), Pai-Irmão $(n=202)$

\begin{tabular}{|c|c|c|c|c|c|c|}
\hline \multirow{3}{*}{ Itens } & \multicolumn{6}{|c|}{ Cargas Fatoriais } \\
\hline & \multicolumn{2}{|c|}{ Mãe-Pai } & \multicolumn{2}{|c|}{ Mãe-Irmão } & \multicolumn{2}{|c|}{ Pai-Irmão } \\
\hline & Afetiv. & Conflito & Afetiv. & Conflito & Afetiv. & Conflito \\
\hline acolhedor & 0,71 & & 0,68 & & 0,68 & \\
\hline afetivo & 0,77 & & 0,62 & & 0,59 & \\
\hline agradável & 0,78 & & 0,67 & & 0,72 & \\
\hline alegre & 0,70 & & 0,60 & & 0,60 & \\
\hline amoroso & 0,72 & & 0,63 & & 0,60 & \\
\hline atencioso & 0,82 & & 0,71 & & 0,73 & \\
\hline carinhoso & 0,68 & & 0,60 & & 0,66 & \\
\hline harmonioso & 0,67 & & 0,72 & & 0,70 & \\
\hline precioso & 0,77 & & 0,62 & & 0,63 & \\
\hline protetor & 0,68 & & 0,75 & & 0,59 & \\
\hline verdadeiro & 0,76 & & 0,58 & & 0,63 & \\
\hline agressivo & & 0,77 & & 0,71 & & 0,59 \\
\hline baixo-astral & & 0,60 & & 0,56 & & 0,44 \\
\hline chato & & 0,68 & & 0,76 & & 0,76 \\
\hline confuso & & 0,68 & & 0,63 & & 0,52 \\
\hline difícil & & 0,81 & & 0,65 & & 0,58 \\
\hline estressante & & 0,77 & & 0,71 & & 0,78 \\
\hline frio & & 0,65 & & 0,60 & & 0,58 \\
\hline nervoso & & 0,75 & & 0,76 & & 0,71 \\
\hline ruim & & 0,72 & & 0,62 & & 0,68 \\
\hline sufocante & & 0,59 & & 0,69 & & 0,76 \\
\hline tenso & & 0,79 & & 0,67 & & 0,56 \\
\hline Alfa de Cronbach & 0,93 & 0,92 & 0,89 & 0,90 & 0,89 & 0,88 \\
\hline Variância Explicada & \multicolumn{2}{|c|}{$55,69 \%$} & \multicolumn{2}{|c|}{$45,55 \%$} & \multicolumn{2}{|c|}{$45,20 \%$} \\
\hline
\end{tabular}

Os resultados descritos na Tabela 2 apontam para a existência de dois fatores para todas as díades, com porcentagem de variância explicada variando de $45,55 \%$ a 55,69\%. As análises com o Alfa de Cronbach tam- bém foram satisfatórias para ambos os construtos. A afetividade apresentou escores de consistência interna variando de 0,89 a 0,93 enquanto o conflito apresentou valores entre 0,89 e 0,92 . 
Após a realização de análises fatoriais exploratórias, procedeu-se a uma análise fatorial confirmatória para dois fatores. Para a confecção do modelo, foram utilizadas como variáveis latentes os construtos afetividade e conflito. Para cada construto foram utilizadas seis variáveis observadas. O construto latente afetividade foi relacionado às variáveis observadas indicadoras de afetividade nas díades participante-pai, participante-mãe, participante-irmão, pai-mãe, pai-irmão e mãe-irmão. Da mesma forma, o construto conflito recebeu os indicadores de conflito destas mesmas díades. Cada variável observada foi obtida por meio da soma dos 11 itens descritos nas Tabelas 1 e 2 . A estratégia de truncar os itens da escala em somente uma variável é amplamente utilizada em análise fatorial confirmatória (Sass \& Smith, 2006) e funciona quando há representatividade e boa consistência entre os itens (Little, Cunningham, Shahar, \& Widaman, 2002). Para a confecção do modelo, foi permitida que as variáveis latentes se correlacionassem. Da mesma forma, foram colocadas correlações entre as percepções de afetividade e conflito das mesmas díades. Deste modo, foi liberada uma correlação entre as variáveis observadas "afetividade participante-pai" e "conflito participante-pai", entre as variáveis "afetividade participante-mãe" e "conflito participante-mãe" e assim por diante para todas as seis díades descritas nas Tabelas $1 \mathrm{e}$ 2. A razão para estas correlações está no fato de que parte da variância do comportamento é específica da díade (vide Kenny, Kashy, \& Cook, 2006 para uma revisão).

Os resultados da análise fatorial confirmatória mostraram relações significativas em quase todas as estima- tivas do modelo. O único valor não significativo foi a correlação entre a afetividade e conflito para a díade participante-pai. A correlação entre as variáveis latentes afetividade e conflito foi negativa e significativa ( $r=-$ $0,41)$. Quanto aos indicadores de adequação do modelo, foi encontrada uma razão $X^{2} / g l(11,63 / 5)$ igual a 2,33 . Os índices Goodness-of-Fit Index (GFI) e o Adjusted Goodness-of-Fit Index (AGFI) foram, respectivamente, de 0,98 e 0,90. Finalmente, o Root Mean Square Error Approximation (RMSEA) apresentou escore de 0,08.

\section{Análises Descritivas e Comparativas dos Escores de Afetividade de Conflito}

Os resultados com as análises do Familiograma não demonstraram nenhuma diferença significativa da afetividade e conflito entre os grupos masculino e feminino. Do mesmo modo, não foi encontrada nenhuma correlação significativa dos construtos com a idade. Comparações entre as estruturas da família indicaram que as crianças e adolescentes do grupo nuclear percebem mais afetividade $(t=4,61, p<0,001)$ e menos conflito $(t=2,66$, $p<0,01)$ na díade mãe-pai quando comparadas à formação não-nuclear. Para as outras díades não foi encontrado resultado significativo.

Tendo em vista a diferença encontrada na percepção do afeto e conflito na díade mãe-pai entre os participantes dos grupos nuclear e não nuclear, optou-se por descrever os dados separadamente para cada um. Na Tabela 3 estão demonstrados os resultados da média, desviopadrão e mediana de todas as díades investigadas nas dimensões afetividade e conflito.

Tabela 3

Médias, Desvio-padrão e Mediana dos Escores de Afetividade e Conflito para as Díades Familiares

\begin{tabular}{llcccc}
\hline \multirow{2}{*}{ Conceito } & \multirow{2}{*}{ Díades } & \multicolumn{4}{c}{ Configuração Familiar } \\
\cline { 3 - 5 } & & \multicolumn{2}{c}{ Nuclear } & Não-nuclear \\
\cline { 3 - 5 } Afetividade & M (DP) & Mediana & M (DP) & Mediana \\
& Participante-mãe & $43,78(8,38)$ & 45,00 & $45,69(8,92)$ & 48,00 \\
& Participante-pai & $44,69(8,55)$ & 46,00 & $43,68(10,85)$ & 47,00 \\
& Participante-irmão & $38,66(10,41)$ & 40,00 & $40,05(12,13)$ & 43,00 \\
& Mãe-pai & $42,91(10,03)$ & 46,00 & $34,47(14,33)$ & 37,00 \\
& Mãe-irmão & $42,58(9,66)$ & 44,00 & $45,46(9,24)$ & 48,00 \\
Conflito & $43,01(9,79)$ & 45,00 & $44,24(10,04)$ & 47,50 \\
& Pai-irmão & $43,03(7,55)$ & 43,91 & $41,71(9,14)$ & 43,25 \\
& Família (Média) & $19,10(7,92)$ & 16,00 & $18,50(7,19)$ & 16,00 \\
& Participante-mãe & $17,75(8,04)$ & 15,00 & $17,22(8,23)$ & 14,00 \\
& Participante-pai & $19,60(7,75)$ & 18,00 & $20,50(10,31)$ & 17,50 \\
& Participante-irmão & $17,65(8,25)$ & 14,00 & $22,41(11,82)$ & 17,00 \\
& Mãe-pai & $17,55(7,54)$ & 14,50 & $17,70(8,41)$ & 15,00 \\
& Mãe-irmão & $16,30(6,57)$ & 14,00 & $17,21(8,79)$ & 13,00 \\
& Pai-irmão & $17,89(6,04)$ & 16,17 & $19,37(8,18)$ & 17,43 \\
& Família (Média) & & & & \\
& & &
\end{tabular}


Após a comparação dos resultados entre os diferentes grupos de participantes, procedeu-se a uma análise da intensidade dos escores de afetividade e conflito entre as díades (participante-mãe, participante-mãe, participante-irmão, mãe-pai, mãe-irmão e pai-irmão). Para isto, foram realizadas análises de variância para medidas repetidas controlada pela configuração da família (nuclear $\mathrm{X}$ não-nuclear). Os resultados apontaram a existência de diferenças significativas tanto na afetividade $(F=6,76$, $p<0,001)$ quanto no conflito $(F=3,83, p<0,01)$, com interação para a configuração familiar.

Análises com teste $t$ para medidas repetidas para o grupo nuclear mostraram que os participantes percebem o relacionamento com o irmão como sendo menos afetivo do que a relação com a mãe $(t=5,80, p<0,001)$ e com o pai $(t=6,13, p<0,001)$. Da mesma forma, a díade fraterna recebeu menor escore de afetividade do que a mãe-pai $(t=3,75, p<0,001)$, mãe-irmão $(t=4,61, p<0,001)$ e paiirmão $(t=4,08, p<0,001)$. Não houve diferença significativa entre as outras díades. Dentro da dimensão conflito, os participantes perceberam a relação pai-irmão como sendo menos conflituosa do que o relacionamento do participante com a mãe $(t=4,21, p<0,001)$, com o pai $(t=3,40, p<0,001)$ e com o irmão $(t=4,47, p<0,001)$. A relação mãe-irmão foi tida como sendo menos conflituosa do que a díade participante-mãe $(t=2,86, p<0,01)$ e participante-irmão $(t=3,08, p<0,01)$.

No grupo não-nuclear, os participantes perceberam menos afetividade na díade mãe-pai, quando comparada com a sua relação com o pai $(t=4,12, p<0,001)$, com a mãe $(t=4,76, p<0,001)$ e a no relacionamento mãe-irmão $(t=3,87, p<0,001)$. Neste grupo, a afetividade do participante com o irmão foi significativamente inferior à do participante-mãe $(t=3,12, p<0,01)$ e mãe-irmão $(t=3,79, p<0,001)$. Já no conflito, a díade mãe-pai possui escores significativamente superiores à do participantepai $(t=3,01, p<0,01)$ e participante-mãe $(t=2,50, p<0,01)$. Também é percebido mais conflito na relação do participante com a mãe do que na da mãe com o irmão $(t=2,73$, $p<0,01)$.

\section{Afetividade, Conflito Familiar e Intensidade dos Sintomas de Depressão}

Os resultados do Familiograma foram comparados com a intensidade dos sintomas depressivos, investigados pelo Inventário de Depressão Infantil. Os resultados do construto afetividade indicaram correlações negativas significativas com a depressão para as díades participantemãe $(r=-0,22, p<0,01)$, pai-irmão $(r=-0,25, p<0,01)$ e mãe-irmão $(r=-0,23, p<0,01)$. A correlação entre a afetividade média da família e a depressão foi de $-0,32$ $(p<0,01)$.

Os resultados das correlações entre o conflito e a intensidade dos sintomas depressivos foram positivos e significativos nas díades participante-mãe $(r=0,27, p<0,001)$, participante-pai $(r=0,33, p<0,001)$, participante-irmão $(r=0,28, p<0,001)$, mãe-irmão $(r=0,33, p<0,001)$ e pai- irmão $(r=0,37, p<0,001)$. O escore médio do conflito familiar também se correlacionou positivamente com a depressão $(r=0,32, p<0,001)$.

No intuito de atribuir uma classificação de família para cada participante (vide Figura 1), foi utilizado o escore da mediana descrito na Tabela 3. Deste modo, o percentil 50 foi o ponto de corte utilizado para classificação das famílias em baixa e alta afetividade e baixo e alto conflito. Setenta e quatro famílias $(31,60 \%$ da amostra total) foram classificadas como sendo do Tipo I (alta afetividade e baixo conflito) e obtiveram média nos escores de depressão de 6,42 pontos $(D P=4,44)$. As famílias do Tipo II (alta afetividade e alto conflito) somaram $36(15,40 \%)$ e tiveram uma média de 11,00 pontos $(D P=6,22)$ nos escores depressivos. Dentre todos os participantes, 41 famílias $(17,50 \%)$ foram classificadas como possuindo baixos níveis de afetividade e de conflito (Tipo III) e apresentaram média de 7,70 pontos nos IDI $(D P=4,77)$. O último grupo familiar possui baixa afetividade e alto conflito (Tipo IV) e possui 83 participantes $(35,50 \%)$ com média de depressão igual a 10,73 pontos $(D P=6,39)$.

Os quatro grupos de famílias foram comparados em seus índices de depressão por meio da Análise de Variância One-Way, que indicou a presença de diferença significativa $(F=8,10, p<0,001)$. Análises post hoc de Bonferroni indicaram que o grupo classificado como Tipo I (alta afetividade e baixo conflito) possui menores escores de depressão do que os Tipos II (alta afetividade e alto conflito) e III (baixa afetividade e alto conflito).

\section{Discussão}

As relações familiares desempenham um importante papel na mediação do funcionamento cognitivo e emocional de seus membros. Neste sentido, são necessárias pesquisas que investiguem tanto o desenvolvimento de instrumentos adequados para a avaliação familiar quanto a correlação destes construtos com sintomas psicopatológicos. O objetivo deste estudo foi o de investigar algumas propriedades psicométricas do Familiograma e a relação dos seus resultados com a intensidade dos sintomas de depressão em crianças e adolescentes. Pretendeu-se desta forma contribuir para uma melhor compreensão das relações familiares com o nível de depressão, ressaltando-se a importância da identificação dos aspectos do sistema familiar que estariam relacionados ao bom funcionamento cognitivo e emocional de seus membros.

Análises fatoriais exploratórias com o Familiograma demonstraram uma estrutura bi-fatorial para todas as representações das díades pesquisadas. Da mesma forma, foi encontrado um padrão claro na disposição das cargas fatoriais dos itens ente os fatores afetividade $\mathrm{e}$ conflito. A porcentagem de variância explicada variou de $42 \%$ a $55 \%$. Os escores de consistência interna foram satisfatórios, variando de 0,87 a 0,93 . Os resultados da análise fatorial confirmatória apresentaram indícios que suportam a natureza bi-fatorial da escala. Estes resulta- 
dos apontam para existência de indícios de validade e fidedignidade do Familiograma para a amostra pesquisada, confirmando os achados descritos em Teodoro (2006) para uma amostra de universitários.

Análises comparativas entre os grupos masculino e feminino não demonstraram diferença significativa quanto aos construtos de afetividade e conflito. Estes resultados estão de acordo com os encontrados por Baptista, Teodoro, Cunha, Santana e Carneiro (2009) em uma pesquisa com universitários e são próximos à baixa correlação encontrada por Herrenkohl, Kosterman, Hawkins e Mason (2009) entre o sexo feminino e conflito familiar $(r=0,10)$. Este conjunto de resultados aponta para uma semelhança na percepção da intensidade destas relações familiares entre os gêneros.

Da mesma forma, não foi encontrada nenhuma correlação significativa da afetividade e conflito com a idade. Alguns autores vêm se utilizando dos processos de individuação, mais especificamente da autonomia e da conectividade, para explicar mudanças ou estabilidade das relações ao longo do ciclo da vida (Youniss \& Smollar, 1985). Com relação à autonomia, existem evidências claras de um aumento da tomada de decisões independentes pelos jovens, o que poderia gerar um maior número de conflitos na relação com os pais (Smetana, 1991). No entanto, com relação à conectividade, que engloba sentimentos como os afetos positivos, negativos e a coesão, não existe um consenso quanto à sua estabilidade ou à sua diminuição (vide, por exemplo, Youniss \& Smollar, 1985). A ausência de correlação entre a idade e a afetividade e conflito sugere uma estabilidade destes construtos do fim da infância para o início da adolescência para a amostra investigada. Deve-se ressaltar, no entanto, que a presente pesquisa possui um delineamento transversal, que não é o mais adequado para a avaliação destas mudanças ao longo do ciclo da vida. Por exemplo, Herrenkohl et al. (2009) encontraram um aumento de conflito familiar em um estudo longitudinal com participantes dos 10 aos 27 anos. A mudança encontrada concentrouse entre a faixa etária dos 14 aos 18 anos. Por outro lado, a ausência de relações significativas na presente pesquisa pode ser devida ao curto intervalo etário investigado (8 a 14 anos), que pode ter contribuído para a ausência de resultados significativos.

Os resultados comparativos entre a percepção de afetividade e conflito pelos participantes de famílias nucleares e não-nucleares mostraram diferenças significativas apenas na relação pai-mãe. Ressalta-se aqui que o uso da terminologia não-nuclear foi adotada devido à heterogeneidade desta sub-amostra, que continha crianças que moravam somente com o pai ou somente com a mãe em decorrência de vários fatores e não somente divórcio. A percepção de maior conflito entre pais que não convivem confirma os resultados encontrados no estudo de Lindsey, Colwell, Frabutt, e MacKinnon-Lewis (2006). Por outro lado, ressalta-se a ausência de diferença signi- ficativa na percepção de afetividade e conflito dos participantes com o pai e mãe entre os grupos nucleares e não-nucleares. Este resultado é interessante, na medida em que estudos de meta-análise vêm mostrando a importância da relação com o pai não-residente para o bemestar e o desenvolvimento emocional do filho (Amato \& Gilbreth, 1999; Dunn, 2004).

As comparações dos escores de afetividade e conflito entre as diversas díades pesquisadas apontaram para uma menor afetividade na relação diádica com o irmão tanto no grupo nuclear quanto no não-nuclear. Este resultado pode ser compreendido pela semelhança de papéis desempenhados pelos irmãos que, apesar do companheirismo, possui também altos níveis de agressão e competição (Dunn, 1983; Turnbull \& Turnbull, 2001).

Análises correlacionais entre as medidas de relacionamento familiar (afetividade e conflito) e a intensidade dos sintomas de depressão indicaram a presença de associações significativas. A afetividade correlacionou-se negativamente, enquanto o conflito está positivamente vinculado a esta psicopatologia. Estes resultados vão ao encontro de pesquisas internacionais, que colocam as relações familiares como estando vinculadas à intensidade dos sintomas depressivos (Galambos et al., 2004; Sheeber et al., 1997).

Com o intuito de comparar a intensidade da sintomatologia depressiva em famílias classificadas como tendo afetividade baixa/alta e conflito baixo/alto, foi elaborada uma classificação de tipos familiares (vide Figura 1). Os resultados indicaram que famílias categorizadas como sendo do Tipo I (alta afetividade e baixo conflito) possuem significativamente menos depressão do que as do Tipo II (alta afetividade e alto conflito) e III (baixa afetividade e alto conflito). Por estes resultados, pode-se perceber que as famílias com os níveis mais elevados de conflito se encontram entre aquelas com maiores níveis de depressão, confirmando o encontrado em outros estudos (Aseltine, Gore, \& Colten, 1998; Pedersen, 1994; Sheeber et al., 1997).

A presente pesquisa pretendeu contribuir para o estudo das propriedades psicométricas do Familiograma e para uma melhor compreensão dos construtos avaliados pelo instrumento com a depressão. De modo geral, foram encontrados índices satisfatórios de validade e fidedignidade do instrumento, além de correlações significativas positivas da depressão com a afetividade e negativas com o conflito. Análises por meio da classificação das famílias em sub-grupos indicaram que o conflito familiar alto aparece associado com a intensidade dos sintomas de depressão percebida por crianças e adolescentes. A utilização desta tipologia mostrou-se uma importante ferramenta, útil tanto na avaliação e quanto na identificação de famílias mais vulneráveis e que possam precisar de uma intervenção clínica. Destaca-se, entretanto, que a presente pesquisa, além de possui um delineamento transversal, é baseada em medidas de auto-relato, o 
que pode ter influenciado as relações significativas encontradas. No intuito de controlar estes efeitos, sugerese a realização de estudos longitudinais e a utilização de múltiplos respondentes em pesquisas futuras.

\section{Referências}

Althoff, R. R. (2008). Diagnoses, neuropsychological functioning, and parental depression affect the expression of internalizing and externalizing disorders in children. Journal of the American Academy of Child \& Adolescent Psychiatry, 4, 358-358.

Amato, P., \& Gilbreth, J. G. (1999). Nonresident fathers and children's well-being: A meta-analysis. Journal of Marriage and the Family, 61, 557-573.

Aseltine, R. H., Gore, S., \& Colten, M. E. (1998). The cooccurrence of depression and substance use in late adolescence. Development and Psychopathology, 10, 549570.

Baptista, M. N., \& Oliveira, A. A. (2004). Sintomatologia de depressão e suporte familiar em adolescentes: Um estudo de correlação. Revista Brasileira de Crescimento Desenvolvimento Humano, 14, 58-67.

Baptista, M. N. Teodoro, M. L. M., Cunha, R. V., Santana, P. R., \& Carneiro, A. M. (2009). Evidência de validade entre o Inventário de Percepção de Suporte Familiar-IPSF e Familiograma - FG. Psicologia: Reflexão e Crítica, 22, 466-473.

Bonomi, A. E., Boudreau, D. M., Fishman, P. A., Meenan, R. T., \& Revicki, D. A. (2005). Is a family equal to the sum of its parts? Estimating family-level well-being for costeffectiveness analysis. Quality of Life Research, 14, 11271133.

Bray, J. H. (1995). Family assessment: Current issues in evaluating families. Family Relations, 44, 469-477.

Byrne, B. M. (1989). A primer of LISREL: Basic applications programming for confirmatory factor analytic models. New York: Springer-Verlag.

Canino, G., Shrout, P. E., Rubio-Stipec, M., Bird, H. R., Bravi, M., Ramirez, R., et al. (2004). The DSM-IV rates of child and adolescent disorders in Puerto Rico: Prevalence, correlates, service use, and the effects of impairment. Archives of General Psychiatry, 61, 85-93.

Dalton, M. A., Adachi-Mejia, A. M., Longacre, M. R., TitusErnstoff, L. T., Gibson, J. J., Martin, S. K., et al. (2006). Parental rules and monitoring of children's movie viewing associated with children's risk for smoking and drinking. Pediatrics, 118, 1932-1942.

Dundas, I. (1994). The Family Adaptability and Cohesion Scale III in a Norwegian Sample. Family Process, 33, 191-202.

Dunn, J. (1983). Sibling relationships in early childhood. Child Development, 54, 787-811.

Dunn, J. (2004). Annotation: Children's relationships with their nonresident fathers. Journal of Child Psychology and Psychiatry, 45, 659-671.

Feinberg, M. E., Button, T. M., Neiderhiser, J. M., Reis, D., \& Hetherington, E. M. (2007). Parenting and adolescent antisocial behavior and depression: Evidence of genotype $\mathrm{x}$ parenting environment interaction. Archives of General Psychiatry, 64, 457-465.

Fleitlich, B., \& Goodman, R. (2001). Social factors associated with child mental health problems in Brazil: A cross sectional survey. British Medical Journal, 323, 599-600.
Galambos, N., Leadbeater, B., \& Barker, E. (2004). Gender differences and risk factors for depression in adolescence: A 4-yearlongitudinal study. International Journal of Behavioral Development, 28, 16-25.

Goodman, A., Fleitlich-Bilyk, B., Patel, V., \& Goodman, R. (2007). Child, family, school and community risk factors for poor mental health in Brazilian school children. Journal of American Academy of Child and Adolescence Psychiatry, 46, 448-456.

Gouveia, V. V., Barbosa, G. A., Almeida, H. J. F., \& Gaião, A. A. (1995). Inventário de Depressão Infantil - CDI: Estudo de adaptação com escolares de João Pessoa. Jornal Brasileiro de Psiquiatria, 44, 345-349.

Hackett, R., \& Hackett, L (1999). Child Psychiatry across cultures. International Review of Psychiatry, 11, 225-235.

Herrenkohl, T. I., Kosterman, R., Hawkins, J. D., \& Mason, W. A. (2009). Effects of growth in family conflict in adolescence on adult depressive symptoms: Mediating and moderating effects of stress and school bonding. Journal of Adolescent Health, 44, 146-152.

Kenny, D. A., Kashy, D. A., \& Cook, W. L. (2006). Dyadic data analysis. New York: The Guilford Press.

Lindsey, E. W., Colwell, M. J., Frabutt J. M., \& MacKinnonLewis, C. (2006). Family conflict in divorced and nondivorced families: Potential consequences for boys' friendship status and friendship quality. Journal of Social and Personal Relationships, 23, 45-63.

Little, T. D., Cunningham, W. A., Shahar, G., \& Widaman, K. F. (2002). To parcel or not to parcel: Exploring the question and weighing the merits. Structural Equation Modeling, 9, 151-173.

McFarlane, A. H., Bellissimo, A., Norman, G. R., \& Lange, P. (1994). Adolescent depression in a school-based community sample: Preliminary findings on contributing social factors. Journal of Youth and Adolescence, 23, 601-620.

Pedersen, W. (1994). Parental relations, mental health and delinquency in adolescents. Adolescence, 29, 975-990.

Pekrun, R. (2001). Familie, Schule und Entwicklung. In S. Walper \& R. Pekrun (Eds.), Familie und Entwicklung. Aktuelle Perspektiven der Familienpsychologie. Göttingen, Germany: Hogrefe.

Phillips, M. R., West, C. L., Shen, Q., \& Zheng, Y. (1998). Comparison of schizophrenic patients' families and normal families in China, using Chinese version of FACES-II and the Family Environment Scales. Family Process, 37, 95-106.

Pinho, C. C. M. (2005). Taxonomia brasileira da personalidade: Um estudo dos adjetivos da língua portuguesa. Tese de Doutorado não-publicada, Programa de Pós-Graduação em Psicologia, Pontifícia Universidade Católica de Campinas, SP.

Rollett, B., \& Werneck, H. (2002). Klinische Entwicklungspsychologie der Familie. Aufgaben und Perspektiven. In B. Rollett \& H. Werneck (Eds.), Klinische Entwicklungspsychologie der Familie. Göttingen, Germany: Hogrefe.

Sass, D. A., \& Smith, P. L. (2006). The effects of parceling unidimensional scales on structural parameter estimates in structural equation modeling. Structural Equation Modeling, 13, 566-586.

Schneewind, K. A. (1999). Familienpsychologie. Stuttgart, Germany: Kohlhammer.

Sheeber, L., Hops, H., \& Davis, B. (2001). Family process in adolescent depression. Clinical Child and Family Psychological Review, 4, 19-35. 
Sheeber, L., Hops, H., Alpert, A., Davis, B., \& Andrews, J. (1997). Family support and conflict: Prospective relations to adolescent depression. Journal of Abnormal Child Psychology, 25, 333-344.

Smetana, J. G. (1991). Adolescents' and mothers' evaluation of justifications for conflicts. New Directions for Children Development, 51, 71-86.

Souza, J. (2008). Filhos de alcoolistas: Afetividade e conflito nas relações familiares. Tese de Doutorado não-publicada, Universidade de São Paulo, Ribeirão Preto, SP.

Teodoro, M. L. M. (2005). Kognitive Repräsentationen familiärer Beziehungen._Methodenkritische Untersuchungen zu Kohäsion und Hierarchie innerhalb des familiären Systems [Representação cognitiva dos relacionamentos familiares. Uma investigação crítico-metodológica da coesão e hierarquia dentro do sistema familiar]. Hamburg, Germany: Kovacs.

Teodoro, M. L. M. (2006). Afetividade e conflito em díades familiares: Avaliação com Familiograma. Interamerican Journal of Psychology, 40, 385-390.

Turnbull, A. P., \& Turnbull, H. R. (2001). Families, professionals and exceptionality: Collaboration for empowerment (4 $4^{\text {th }}$ ed.). Upper Saddle River, NJ: Merrill.

Wathier, J. L., Dell'Aglio, D. D., \& Bandeira, D. R. (2008). Análise fatorial do Inventário de Depressão Infantil (CDI) em amostra de jovens brasileiros. Avaliação Psicológica, 7, 75-84.

Windle, M. (1992). A longitudinal study of stress buffering for adolescent problem behaviors. Developmental Psychology, 28, 522-530.

Youniss, J., \& Smollar. J. (1985). Adolescent relations with mothers, fathers, and friends. Chicago: University of Chicago Press. 